OVX group. In the TPTD and ALN group, the expression levels of TNF- $\alpha$ was significantly degreased than the OVX group. And the expression levels of TNF- $\alpha$ was no significance between the TPTD and ALN group.

Conclusions: In this study, TPTD and ALN treatments prevented bone loss in OVX mice. Mechanical hyperalgesia in hindlimbs tended to be decreased in the OVX group compared with the TPTD and ALN group. ALN treatment was more effective in bone formation compared with TPTD treatment, whereas pain relief was no significance between TPTD and ALN treatment. These results suggest that TPTD treatment was more effective in osteoporosis patients with skeletal pain.

Disclosure of Interest: None declared

DOI: 10.1136/annrheumdis-2017-eular.4628

\section{FRI0567 DIFFERENCES IN ADCHERENCE TO OSTEOPOROSIS MEDICATIONS IN PATIENTS WITH RHEUMATIC DISEASES: A 3-YEAR RETROSPECTIVE COHORT STUDY}

O. Nikitinskaya, N. Toroptsova. Nasonova Research Institute of Rheumatology, Moscow, Russian Federation

Background: Patients with rheumatic diseases (RD) have an increased risk of developing osteoporosis (OP) compared with healthy population due to chronic inflammation, low physical activity and using some kind of medications. Persons with $\mathrm{RD}$ and $\mathrm{OP}$ require prolonged osteoporosis treatment due to increased risk of fracture.

Objectives: To evaluate adherence and persistence with different osteoporosis medications in RD patients with OP during 3 years.

Methods: We conducted a retrospective study of 204 patients $(82 \%$ women, mean age $54 \pm 11$ years) with RD (93 - rheumatoid arthritis, 48 - systemic sclerosis, 39 - systemic lupus erythematosus and 24 - ankylosing spondylitis), whom OP was diagnosed in 2013. In 2016 we revised there medical charts for collection information on the osteoporosis treatment and performed telephone contact for patient's self-reporting.

Results: 196 (96\%) patients started the OP treatment. Among them 26\% alendronate, $24 \%$ received alfacalcidol or supplements of calcium and vitamin D only, $16 \%$ - stroncium ranelate, $12 \%$ - ibandronate, $9 \%$ zoledronic acid, $8 \%$ calcitonin and $5 \%$ - denosumab.

$8(4 \%)$ patients didn't start the drug treatment at all, $72(35 \%)$ patients were persisted less than 12 months, $47(23 \%)-2$ years and $77(38 \%)-3$ years. Mean persistence was $2.1,2.4,1.6,1.7,1.9,2.0$ years for alendronate, alfacalcidol, stroncium ranelate, ibandronate, zoledronic acid, denosumab, respectively. 61 (31\%) persons were switched from one antiosteoporotic medication to another due to side effects, inconvenient dose regimen or other reasons. Persistence to oral therapy was better among women than men $(p<0.05)$, especially on daily regimen. Discontinuers were less likely than persistent subjects to visit rheumatologist and BMD measurement each year for control of treatment $(p<0.05)$. Patients with ankylosing spondylitis were less persistent than persons with other RD. Presence of OP fractures in anamnesis had no significant influence on adherence. 8 (4\%) patients had fractures during retrospective period, among them 6 - received only supplements of calcium and vitamin D, 1 - zoledronic acid once and 1 - without OP therapy

Conclusions: Only $1 / 3$ of patients with RD received antiosteoporotic drugs during 3 years. Visits to rheumatologist and BMD measurements increased subject's persistence. Improving the quality of medical care can be achieved through education and motivation of patients for a long-term treatment of OP.

Disclosure of Interest: None declared

DOI: 10.1136/annrheumdis-2017-eular.2422

\section{FRI0568 ECHOSOUND APPROACH FOR SHORT-TERM FOLLOW-UP OF THE DENOSUMAB EFFECT ON BMD RECOVERY AGAINST AROMATASE INHIBITOR IMPACT IN BREAST CANCER PATIENTS}

P. Pisani ${ }^{1}$, M. Muratore ${ }^{2}$, F. Conversano $^{1}$, E. Casciaro ${ }^{1}$, M. Di Paola ${ }^{1}$, R. Forcignanò ${ }^{3}$, M. Ciccarese ${ }^{3}$, G. Surico ${ }^{3}$, L. Quarta ${ }^{2}$, E. Quarta ${ }^{2}$,

D. Costanza ${ }^{2}$, R. Franchini ${ }^{1}$, R. Tarparelli ${ }^{4}$, S. Casciaro ${ }^{1} .{ }^{1} 1$ National Research Council, Institute of Clinical Physiology; ${ }^{2} 2 \mathrm{OU}$ of Rheumatology Galateo Hospital, San Cesario di Lecce, ASL-LE; ${ }^{3} 30 U$ of Oncology Vito Fazzi Hospital, ASL-LE; ${ }^{4}$ E Echolight Spa, LECCE, Italy

Background: The Aromatase Inhibitors (Als)-based therapy used in breast cancer patients to profoundly lower estrogen levels seems to enhance the loss of bone mineral density (BMD) and to increase the fragility fracture rate [1]. Several clinical studies demonstrated that, in breast cancer patients that received the adjuvant Als, the subcutaneous administration of Denosumab-based therapy significantly increased BMD values and reduced the rate of clinical fractures.

Objectives: To monitor the short-term Denosumab and Als therapeutic effects on BMD in breast cancer patients through an innovative echographic approach, the EchoSound technology [2]

Methods: 154 breast cancer patients selected for receiving the adiuvant Als therapy were recruited. All the patients underwent spinal and femoral dual X-ray absorptiometry (DXA) examinations before Als therapy administration starting (time T0). After Als treatment starting, enrolled patients were divided into 2 groups:
105 patients received only the Als treatment (Group A), whereas the remaining 49 patients (Group B) received also an additional Denosumab treatment, in order to contrast the BMD reduction induced by Als administration. Follow-up measurements were conducted at two different time points: 12 (T1) and 18 (T2) months from Als treatment starting. At time T1, patients underwent both DXA examinations and EchoSound echographic scans, whereas at time T2 only the echographic scans were performed, since DXA cannot be used for short-term follow-ups.

Results: At time T1, the following results were obtained on lumbar spine: Group A showed a BMD decrement, which was equal to $-2.07 \% \pm 1.66 \% \quad(p<0.01)$ according to DXA and to $-2.22 \% \pm 0.89 \%(p<0.01)$ according to EchoSound; Group B showed a BMD increase of $4.06 \% \pm 1.49 \%(p<0.01)$ and $4.31 \% \pm 0.62 \%$ $(p<0.01)$ as measured by DXA and EchoSound scans, respectively. At time T2, Group A showed a further BMD decrement, resulting in a total decrease of $-3.95 \% \pm 1.09 \%(p<0.01)$ with respect to T0 values; on the contrary, in Group $B$ Denosumab treatment produced an additional BMD increment, resulting in a total BMD increase of $4.98 \% \pm 1.03 \%(p<0.01)$ in the same 18 -month period. Similar results were obtained for femoral neck BMD: a total BMD decrease of $-2.37 \% \pm 0.97 \%(p<0.01)$ during the whole treatment period was observed in Group A, whereas a total BMD increment of $3.53 \% \pm 0.43 \%(p<0.01)$ was measured in the same period in Group B.

Conclusions: By using the EchoSound technology the short-term follow-up of the positive Denosumab effects on BMD reduction in patients treated with adjuvant Als was feasible and accurate. This approach can be also useful to monitor the therapy effectiveness in patients undergoing specific anti-osteoporotic treatments. References:

[1] J Clin Endocrinol Metab.2011;96:308.

[2] Clin Cases Min Bone Metab 2015;12:142.

Acknowledgements: Work partially funded by FESR PO Apulia Region 2007-13; Action 1.2.4 (grant n. 3Q5AX31: ECHOLIGHT Project).

Disclosure of Interest: P. Pisani: None declared, M. Muratore: None declared, F. Conversano Shareholder of: Echolight spa, a National Research Council spin-off that may or may not benefit from results of this study, E. Casciaro Shareholder of: Echolight spa, a National Research Council spin-off that may or may not benefit from results of this study, M. Di Paola: None declared, R. Forcignanò: None declared, M. Ciccarese: None declared, G. Surico: None declared, L. Quarta: None declared, E. Quarta: None declared, D. Costanza: None declared, R. Franchini: None declared, R. Tarparelli Employee of: Echolight Spa, S. Casciaro Shareholder of: Echolight spa, a National Research Council spin-off that may or may not benefit from results of this study

DOI: 10.1136/annrheumdis-2017-eular.4060

\section{FRI0569 EVALUATION OF BONE MICROARCHITECTURE IN SYSTEMIC SCLEROSIS PATIENTS: RELATIONSHIPS BETWEEN TRABECULAR BONE SCORE (TBS) AND DISEASE SEVERITY}

S. Paolino ${ }^{1}$, E. Gotelli ${ }^{1}$, A. Sulli ${ }^{1}$, B. Ruaro ${ }^{1}$, M. Ghio ${ }^{1}$, C. Pizzorni ${ }^{1}$, E. Alessandri ${ }^{1}$, V. Tomatis ${ }^{1}$, C. Seriolo ${ }^{2}$, L. Molfetta ${ }^{2}$, M. Cutolo ${ }^{1}$, A. Casabella ${ }^{1}$ ${ }^{1}$ Department of Internal Medicine, University of Genova, IRCCS AOU San Martino, Genoa, Italy, Research Laboratory and Academic Division of Clinical Rheumatology, Department of Internal Medicine, University of Genova, Genoa, Italy; ${ }^{2}$ Department of Internal Medicine, University of Genova, Genoa, Italy, Osteoporosis, Bone and Joint Disease Research Center, CROPO, Genoa, Italy

Background: Systemic sclerosis (SSc) is a rare connective tissue disorder characterized by an increased synthesis and deposition of extracellular matrix in the skin and internal organs (1). Several studies described SSc as potential risk factor for osteoporosis, however, to date the bone quality in SSc is unclear (2). Trabecular bone score (TBS) has been recently proposed as an indirect measure of bone microarchitecture (3).

Objectives: The aim of this study was to assess bone microarchitecture in SSc patients and possible association with disease severity and microangiopathy. Methods: Twenty-three female SSc patients (mean age 63.2 \pm 12.8 SD years mean disease duration $92.8 \pm 66$ SD months, mean Raynaud's Phenomenon duration 142.6 \pm 126.1 SD months) were enrolled after written informed consent. The assessment of disease severity was performed using the Medsger's severity scale (4). Bone Mineral Density (BMD) measurements at L1-L4, femoral neck and total hip, were performed using DXA Prodigy Densitometer (GE Lunar). TBS was derived for each spine DXA examination using the TBS index (TBS iNsight Medimaps). Nailfold videocapillaroscopy (NVC) was used to assess the microangiopathy based on nailfold video capillaroscopic pattern (NVC) analysis and the microangiopathy evolution score (MES) (5-6). Using the FRAX (Fracture Risk Assessment Tool) we also evaluate the 10-year risk of hip and major joints osteoporotic fracture.

Results: A positive correlation was observed between TBS and Medsger's general organ score $(r=0.5 ; p=0.01)$; no other correlations were found between TBS and Medsger's score. Interestingly, TBS was positively and significantly correlated with modified Rodnam skin score (mRss) $(p=0.01)$. When the patients were divided in two groups considering skin involvement by mRss, TBS was found significantly higher into the group with $\mathrm{mRss}>15$ compared to the group with $\mathrm{mRss}<15(1.255 \pm 0.08$ vs $1.163 \pm 0.03 ; \mathrm{p}=0.01)$ No correlations were found between NVC patterns/MES and bone quality assessment (TBS) or bone density assessment (BMD), only a significant correlation, as expected, was observed 
between MES and skin involvement (mRss) $(\mathrm{p}=0.05)$. On the other hands, FRAX, the major osteoporotic fracture risk, positively correlates with Medsger's kidney disease severity $(p=0.04)$ and Medsger's lung disease severity $(p=0.04)$; in addition, FRAX, for hip fracture risk, seems to correlate significantly with Medsger's lung involvement severity $(p=0.04)$.

Conclusions: This study demonstrates in SSc patients a relationship between clinical disease severity (organ fibrosis/failure) and altered bone microarchitecture (TBS). In addition, skin involvement was found significantly correlated with altered quality of the trabecular bone architecture (TBS) and a significant increase of osteoporotic fracture risk (FRAX) was found correlated with kindey and lung involvement.

References:

[1] Varga J, Abraham D. J Clin Invest. 2007 Mar;117(3):557-67.

[2] Loucks J, Pope JE. Semin Arthritis Rheum. 2005 Feb;34(4):678-82.

[3] Silva BC et al. J Bone Miner Res. 2014 Mar;29(3):518-30.

[4] Medsger TA et al. Clin Exp Rheumatol. 2003;21(3 Suppl 29):S42-6.

[5] Sulli A, et al. Ann Rheum Dis 2008;67:885-7.

[6] Cutolo et al. J Rheumatol 2000; 27:155.60.

Disclosure of Interest: None declared

DOI: 10.1136/annrheumdis-2017-eular.6415

\section{FRI0570 OSTEOPOROSIS AND BREAST CANCER: OUTCOMES AT A SPECIALIZED OSTEOPOROSIS CLINIC FOLLOWING A STRUCTURED ASSESSMENT}

S. Lopez-Salguero $^{1}$, J.C. Ordoñez ${ }^{1}$, L. Ranieri ${ }^{1}$, M. Andrés ${ }^{1,2}$, J. Ponce $^{3}$, I. Ibero ${ }^{1} .{ }^{1}$ Rheumatology, Hospital General Universtiario Alicante;

${ }^{2}$ Departamento de Medicina Clínica, Universidad Miguel Hernández de Elche;

${ }^{3}$ Oncology, Hospital General Universtiario Alicante, Alicante, Spain

Background: Women with breast cáncer (BC) are at risk for the development of bone loss and osteoporosis (OP) mainly due to adjuvant therapies. Aromatase inhibitors (Al) therapy fully suppresses estrogen synthesis, further exacerbating the increased bone resorption and leading to an excess fracture risk. Thus, a close monitoring of bone mineral metabolism is recommended.

Objectives: The aim of the present study was to analyze bone health status and clinical characteristics of women $\mathrm{BC}$ referred by oncologist to a specialized clinic and their outcomes during follow-up.

Methods: Retrospective analysis of consecutive female patients with recent BC and low bone mineral density (BMD) referred to the osteoporosis outpatient clinic for assessment, as agreed with oncologists. A descriptive analysis of epidemiological, clinical, laboratory, imaging, and dual energy $x$-ray absorptiometry (DEXA) data is presented, both at baseline and last visit. $95 \%$ confidence intervals $(95 \% \mathrm{Cl})$ were estimated for rate of fragility fractures (FF) at baseline and during follow-up. Results: A total number of 156 female patients have been assessed up to January 2017; median aged $60.7(\mathrm{SD} \pm 10.6)$ years old, $89 \%$ postmenopausal. BC was mainly non-methastasic $(117 ; 75 \%)$, and $110(70 \%)$ patients were on aromatase inhibitors (14 on anastrozole, 89 on letrozole, and 7 on exemestane). 36 patients $(23 \%)$ were active smokers, and $17(11 \%)$ had stopped smoking. At baseline, 37 patients $(24.2 \%, 95 \mathrm{Cl} \% 20-37)$ had previous FF, mostly vertebral (19) or non-vertebral (13), and three cases had suffered from multiple FF. BMDs were at osteoporotic range at the lumbar spine (median T-score -2.6 p25/75 -2.2, -3.2) and osteopenic at both the femoral neck and hip (mediant T-score -1.9 p25/75 -1.1, -2.3). Regarding antiosteoporotic therapies, bisphosphonates were prescribed in 110 cases $(70.5 \%)$, denosumab in $24(15.3 \%)$, and raloxifene in two cases $(1.2 \%)$; in the others $(12.8 \%)$ only calcium plus vitamin D supplementation was recommended. A total of 107 patients were followed a median of 2.13 years (p25-75 1.23-3.18). During follow-up, new FF occurred in 13 patients $(12.1 \%$, $95 \% \mathrm{Cl} 6-19$ ), that were vertebral in 8 , non-vertebral in 4 , both in one case, while no hip FF were detected.

Conclusions: outcomes of a structured assessment of female patients with BC and low BMD are reported here. Despite this, $12 \%$ of cases developed a new $\mathrm{FF}$, highlighting the need for special attention to this singular, secondary form of osteoporosis.

Disclosure of Interest: None declared

DOI: 10.1136/annrheumdis-2017-eular.4928

\section{FRI0571 OSTEOPOROSIS AND BREAST CANCER: CAN FRAX-BASED RISK FACTORS ACCURATELY PREDICT FURTHER FRACTURES AT THIS SETTING?}

S. Lopez-Salguero $^{1,1}$, J.C. Ordoñez ${ }^{1}$, L. Ranieri ${ }^{1}$, M. Andrés ${ }^{1,2}$, J. Ponce ${ }^{3}$, I. Ibero ${ }^{1} .{ }^{1}$ Rheumatology, Hospital General Universtiario Alicante;

${ }^{2}$ Departamento de Medicina Clínica, Universidad Miguel Hernández de Elche;

${ }^{3}$ Oncology, Hospital General Universtiario Alicante, Alicante, Spain

Background: Women with breast cáncer (BC) are at risk for the development of bone loss and osteoporosis (OP) mainly due to adjuvant therapies, as aromatase inhibitors (Al). Thus, it would be of special interest in this group of patientes, to know at baseline wich factors can predispose to develope fragility fractures (FF) during follow-up, in order to optmize vigilance and treatment.

Objectives: The purpose of this study is to analyze wich risk factors at baseline that can predict the appereance of a new FF in women with $\mathrm{BC}$ and $\mathrm{OP}$.
Methods: Retrospective analysis of consecutive female patients with recent breast cancer (BC) and low bone mineral density (BMD) referred to the osteoporosis outpatient clinic for assessment, as agreed with oncologists. Fort he purpose of this anaylisis, only patients with follow-up data (at least six months after baseline visit) were selected. FRAX tool [1]-derived risk factors (age, BMI, DEXA, previous fracture, parent fractured hip, smoking, alcohol, glucocorticoids, rheumatoid arthritis, secondary OP) were taken as explicative variables. Student's $t$ and chi-2 tests were used to perform comparisons base don the appereance of new FF in the study period.

Results: A total number of 156 female patients have been assessed up to January 2017. Of the 107 patients in follow-up (68.5\%; median time in follow-up 2.1 years p25-75 1.2-3.2). Median age was 62.07 years old $(S D \pm 10,35)$, being $89 \%$ of them postmenopausal. $73(68,2 \%$ ) were on Al therapy (10 anastrozole, 59 letrozole and 4 exemestane). At baseline, 29 patients (27.1\%) showed a FF (15 vertebral; 10 non vertebral; 2 hip; 2 multiple fracture). Antiosteoporotic treatment was recommended in 95 patients $(88.7 \%)$. During follow-up, $13 \mathrm{FF}$ were seen $(12,1 \%$; Cl95\% 6-19); being 8 of them vertebral, 4 not vertebral, and one multiple fracture; no new hip FF was seen.

After comparison of the different risk factors according to the development of a new FF, no significant association was found (see table).

Table 1

\begin{tabular}{|c|c|c|c|}
\hline & \multicolumn{2}{|c|}{ New fragility fracture } & \multirow[t]{2}{*}{$\mathrm{p}$} \\
\hline & $\mathrm{NO}(\mathrm{n}=94)$ & YES $(n=13)$ & \\
\hline Age (years old), mean $\pm S D$ & $62.0 \pm 10.7$ & $62.1 \pm 9.9$ & 0.956 \\
\hline $\mathrm{BMI}\left(\mathrm{kg} / \mathrm{m}^{2}\right)$, mean $\pm \mathrm{SD}$ & $26.5 \pm 7.1$ & $23.1 \pm 10.0$ & 0.213 \\
\hline Follow-up duration (months) median $\pm \mathrm{SD}$ & $28.6 \pm 19,4$ & $30.6 \pm 12.8$ & 0.723 \\
\hline DEXA at lumbar spine (T-score) median $\pm S D$ & $-2.7 \pm 0,8$ & $-2.9 \pm 0.6$ & 0.256 \\
\hline $\mathrm{GFR}(\mathrm{m} / \mathrm{min}) \mathrm{mean} \pm \mathrm{SD}$ & $91.7 \pm 16.0$ & $97.2 \pm 11.2$ & 0.282 \\
\hline Menopause (\%) & 93.4 & 91.6 & 0.822 \\
\hline Previous fracture (\%) & 27.1 & 38.4 & 0.512 \\
\hline Parent fractured hip (\%) & 11.0 & 25.0 & 0.125 \\
\hline Smoking (\%) & 10.0 & 8.3 & 0.721 \\
\hline Glucocorticoids (\%) & 9.9 & 7.7 & 1.000 \\
\hline Rheumatoid arthritis (\%) & 3.2 & 0 & 1.000 \\
\hline Aromatase inhibitor (\%) & 70.9 & 69.2 & 0.897 \\
\hline Antiosteoporotic treatment (\%) & 88.4 & 92.3 & 0.676 \\
\hline
\end{tabular}

Conclusions: In this study no relationship between FRAX-based risk factors and the development of new FF in women with OP and BC was found. As new FF occurred in $12 \%$ of cases, it highlights the need for special attention to this singular, secondary form of OP.

References:

[1] Kanis JA, Johnell O, Oden A, Jonsson B, Dawson A, Dere W. Risk of hip fracture derived from relative risks: an analysis applied to the population of Sweden. Osteoporosis International 2000; 11: 120-127.

Disclosure of Interest: None declared

DOI: 10.1136/annrheumdis-2017-eular.5211

\section{FRI0572 IDENTIFYING WOMEN AND MEN AT HIGH FRACTURE RISK BY LEVERAGING THE ELECTRONIC MEDICAL RECORDS TO ESTIMATE FRAX TREATMENT THRESHOLDS}

\section{S. Amin, E. Atkinson, S. Khosla. Mayo Clinic, Rochester, United States}

Background: In the busy clinic, identifying individuals at high fracture (fx) risk who warrant intervention can be a challenge. There are several medical conditions that increase the risk for bone loss and falls which are recorded in the electronic medical record (EMR).

Objectives: We explored whether we could exploit data available in the EMR to estimate FRAX treatment thresholds to help passively identify patients who would benefit from further bone mineral density screening and management.

Methods: We studied 912 women and men, previously recruited for our bone health studies, in whom FRAX scores (with and without BMD) had been determined and comprehensive medical diagnoses were available through the medical linkage system of the Rochester Epidemiology Project. All diagnoses were categorized by the Clinical Classification Software (CCS) system whereby over 14,000 ICD-9-CM diagnoses are reduced to 568 clinically meaningful categories. If a subject had at least two diagnoses in a CCS category that were at least 30 days apart and within 5 years of their FRAX assessment, the subject was treated as having that CCS code. We used Gradient Boosting Machine (GBM) to create models that would predict the treatment thresholds for the FRAX 10-year risk for major osteoporotic (OP) fx (>20\%) and hip fx (>3\%), based on available diagnoses. Models were fit using age, sex and CCS category from $80 \%$ of the data, retaining $20 \%$ for validation.

Results: Of the $564(62 \%)$ women and $348(38 \%)$ men, the mean \pm SD age was $61 \pm 16 \mathrm{yrs}$. There were no significant differences in subject characteristics used for FRAX calculation or FRAX scores between the training and validation sets. The c-statistic for GBM models predicting treatment thresholds for FRAX, calculated with BMD, for major OP fx and hip fx were 0.95 and 0.96 , respectively, for the training set, and 0.88 and 0.94 for the validation set. Similar results were observed for FRAX scores without BMD.

Conclusions: FRAX treatment thresholds may be reasonably estimated from data available in the EMR to help identify to the clinician those at highest risk 